\title{
Introducción histórica a la docencia de la Documentación en los estudios de Periodismo
}

\author{
A historical introduction to Library and Information Science education in Journalism studies
}

\author{
Rubén Domínguez-Delgado (1), Francisco-Javier MuÑoz-GaRcía (2), \\ María-Ángeles LóPEZ-HERNÁNDEZ (1)
}

(1) Universidad de Sevilla, Facultad de Comunicación, Avenida Américo Vespucio, s/n, 41092, Sevilla, rdd@us.es, alhernan@us.es (2) Universidad de Huelva, Facultad de Psicología, Avenida de las Fuerzas Armadas, s/n, 21007, Huelva, fjgarcia@uhu.es

\begin{abstract}
Resumen
Realizamos un recorrido histórico de casi un siglo por los estudios de Periodismo en España, desde que se crea la primera escuela de periodismo, la del diario El Debate, en 1926, hasta los grados en periodismo ofertados en las universidades públicas en el curso 2020/2021, con el fin de analizar el nivel de presencia e importancia que ha tenido la docencia de la Documentación en tales estudios, dada la inexistencia de un trabajo similar que aglutine todo este espectro histórico de casi un siglo. En lo que respecta a la etapa universitaria, que se inicia en 1971 y en cuyo análisis reside la principal aportación de este trabajo, caben destacar momentos álgidos, fundamentalmente en la última década del siglo XX y en los primeros años del $X X I$, seguidos de una progresiva y aún vigente fase de declive, principalmente desde finales de la primera década del presente siglo, tras la adaptación al Espacio Europeo de Educación Superior, que ha traído consigo, por lo general, una reducción en el número de asignaturas vinculadas a la Documentación ofertadas en Periodismo y de horas de formación en dicha disciplina, especialmente de carácter básico y obligatorio. En el momento actual, se observan diferencias sustanciales a nivel nacional entre las ofertas de asignaturas de Documentación dentro del Grado en Periodismo, oscilando estas materias entre tres y una, con disparidad en cuanto a sus números de horas formativas, su carácter y el curso en el que se programan.
\end{abstract}

Palabras clave: Documentación periodística. Archivos de prensa. Periodismo. Documentación. Docencia. Educación. Planes de estudio. España.

\section{Introducción}

La formación universitaria en Periodismo en España en universidades legalmente reconocidas (1) no comenzó hasta 1971, cuando abrió el primer centro universitario de periodismo bajo el nombre de Facultad de Ciencias de la Información, en la Universidad Complutense de Madrid. Su primer plan de estudios, regulado por el Ministerio de Educación y Ciencia (1971), ya incluía un curso específico denominado Documentación. Desde ese momento hasta la actualidad, la disciplina científica de la Documentación ha estado siempre presente, aunque desde diferentes enfoques o

\begin{abstract}
A historical journey of almost a century is made through the studies of Journalism in Spain, since the creation of the first journalism school, that of the newspaper El Debate, in 1926, to the current Bachelor's Degrees in Journalism which are taught in Spanish public universities for the academic year 2020-2021, in order to analyze the level of presence and importance of Library and Information Science (LIS) on these studies, given the lack of a similar work that brings together this entire historical spectrum of almost a century. Regarding the university stage, which began in 1971 and in whose analysis lies the main contribution of this work, there have been moments, mainly in the last decade of the twentieth century and in the first years of the twenty-first, followed by a progressive and still in force period of decline, especially since the end of the first decade of the current century, after the adaptation of university studies to the European Higher Education Area, which has generally brought with it a reduction in the number of subjects linked to LIS offered in Journalism studies and of hours of training in said discipline, especially of a basic and mandatory nature. Nowadays, considerable differences are observed at the national level among the offers of LIS subjects within the Bachelor's Degree in Journalism, these courses oscillating between three and one, with disparity in terms of their number of training hours, their nature and the year in which they are programmed.
\end{abstract}

Keywords: Journalism documentation. News archives. Journalism. Library and Information Science. Teaching. Education. Curriculum. Spain.

perspectivas, en la mayoría de los planes de estudios universitarios en periodismo, con asignaturas específicas. Pero esta larga tradición de la disciplina documental en la formación universitaria española en periodismo ya contaba con una serie de antecedentes históricos en las escuelas de periodismo preuniversitarias, que comienzan su andadura en 1926 con la creación de la del diario El Debate, la pionera (Cantavella, 2017).

En la presente investigación nos planteamos como principal objetivo realizar un recorrido histórico por los orígenes y evolución de la docencia de cursos o materias vinculadas a la disciplina 
científica de la Documentación en los diversos y cambiantes estudios de Periodismo en España, desde que se crea la citada primera escuela en 1926 hasta la formación del Grado en Periodismo ofertada en el curso académico 2020/2021 por las universidades públicas españolas, dada la ausencia de un estudio de estas características que aglutine todo el espectro temporal, de casi un siglo, de formación en periodismo en nuestro país. Nos detenemos especialmente en la más reciente etapa universitaria, que se inicia en 1971 y en cuyo análisis reside la principal aportación de este trabajo. La descripción evolutiva en el ámbito formativo se acompaña, para una mayor contextualización, de algunos hitos importantes en el campo del periodismo, la documentación y la educación en nuestro país.

Se empleó el método de análisis cualitativo diacrónico de revisión bibliográfica de los documentos oficiales reguladores de los estudios de periodismo en España, así como de literatura gris, planes docentes y programas o guías de materias ofertadas hasta el curso académico 2020/2021 y, por supuesto, de literatura científica, en relación con los campos de la documentación, el periodismo y la educación.

Es preciso aclarar que la última etapa de la investigación dedicada a los estudios universitarios de Grado, a los que se adaptaron las universidades españolas entre 2008 y 2010 , conforme al Espacio Europeo de Educación Superior - sobre la cual radica la principal aportación de este trabajo- se centró, exclusivamente, en las universidades públicas españolas y en la titulación oficial denominada Grado en Periodismo. Así, no se abarcan en este estudio, desde ese momento temporal, las cada vez más numerosas universidades privadas, así como tampoco las crecientes dobles titulaciones y los grados híbridos (como es el caso, por ejemplo, del Grado en Comunicación y Periodismo Audiovisuales que oferta la Universidad de Lleida).

\section{La Documentación en las primeras escuelas de periodismo preuniversitarias}

Como apuntábamos al inicio, antes de 1971 había ya en España una formación en Periodismo, aunque no universitaria. La primera escuela española de Periodismo fue creada en Madrid en 1926 por iniciativa de la Iglesia, la Asociación Católica de Propagandistas y el diario El Debate (Cantavella, 2017), liderados por Ángel Herrera Oria. Este medio impreso católico había creado también, en 1917, el considerado por Tapia (1995, p. 240) como uno de los primeros centros de documentación en un medio periodístico español (junto con el de La Vanguardia, que nace en 1920), que se gestó en paralelo a la primera hemeroteca pública del país, la Hemeroteca Municipal de Madrid, inaugurada en 1916.

López Yepes atribuye a Manuel Graña González (1978, p. 312), uno de los impulsores de la escuela de El Debate, "un primer esbozo de las misiones del centro de documentación de la empresa periodística y, correlativamente, la necesidad de su enseñanza en el ámbito de la formación del periodista". Y es que, en el diseño del programa formativo del segundo de los cuatro períodos académicos de las enseñanzas en El Debate, Graña González (1930, p. 48) -quien durante dos años había realizado un estudio en Estados Unidos sobre la formación en periodismo en sus universidades y escuelas-, incluyó: "Libros y estadísticas y documentos escritos que pueden ser fuentes de información. Archivo del periódico. Biblioteca del redactor. Manera de utilizar estas fuentes con el menor esfuerzo posible". Y también incorporó en el de cuarto curso el "estudio de los fondos de los principales periódicos" (ibidem, p. 49). Además, la lección XXXIX, de las cuarenta y tres recogidas en el leccionario diseñado por el propio Graña González (ibidem, p. 137), se dedica a los temas: "Documentación próxima y remota. Los periódicos como documentos. Libros de consulta. Registro de noticias y opiniones. Criterio y reflexión. Por lo que respecta a las fuentes de información".

Aunque proporcionaba una formación privada y profesional, fuera del ámbito estrictamente académico, la escuela de El Debate originó una discusión en aquella época sobre la posibilidad de tener una formación universitaria en periodismo en España. Pero estas iniciativas, así como la actividad de la escuela y del propio diario, fueron interrumpidas con el estallido de la Guerra Civil en 1936.

Franco, ya bajo su dictadura, aprobó en 1938 una Ley de Prensa para controlar los medios de comunicación y, al mismo tiempo, creó en Madrid la primera Escuela Oficial de Periodismo, que inició su formación en 1942. Desde el comienzo de su andadura, hubo varias referencias a la disciplina de la Documentación dentro de los programas de algunos cursos impartidos en esta nueva escuela (Tapia, 1995, pp. 234-238), gracias a un grupo de profesores que reivindicaron la importancia para los estudiantes y futuros periodistas de tener una formación en esta ciencia.

Así, por ejemplo, Bartolomé Mostaza, profesor del curso Información y reportajes, incluyó desde 1942, en su lección 28, el contenido Periodismo indirecto: recoger, cribar, comprobar. El archivo y su manejo. El profesor de la materia Periodismo Gráfico, Mario Rodríguez, incluyó en 1955, en su lección 30 , una clase sobre archivos fotográficos 
y sus criterios de selección y clasificación. Además, Enrique De Aguinaga incluyó en 1961, dentro de su curso Seminario de Redacción, temas como Administración de datos y antecedentes y Documentación previa. Y el profesor Luis Fernando Bandín incluyó, en 1963, dentro del programa de su curso Técnicas del Periodismo Impreso, un sexto tema denominado El archivo. Su organización. Una redacción orientada hacia el pasado en función de la actualidad inmediata.

Por otro lado, desde el curso académico 1965/1966, el profesor Ramón Fernández impartiría en la Escuela Oficial de Periodismo el seminario Curso Monográfico de Hemerografía —denominado más tarde Normas de Archivo y de Análisis Hemerográfico-. Algunos de los objetivos de este curso eran la formación para documentalistas de prensa o el aprendizaje de la estructura y las dinámicas dentro de los servicios de documentación modernos -archivos de prensa, bibliotecas, archivos fotográficos, archivos sonoros-

Pero tras la aprobación de una nueva Ley de Prensa en España en 1966, sería reconocido un nuevo plan de estudios para la Escuela Oficial de Periodismo en 1967, que incorporaba muchos más cursos y nuevas disciplinas y que reconocía los estudios de periodismo como estudios superiores, de 4 años de duración. Fue entonces cuando se incluiría por primera vez un curso específico independiente de Documentación: $\mathrm{He}$ merografía y técnicas de documentación informativa, impartido en segundo curso una vez a la semana, por el profesor Ramón Fernández (BOE, 1967, p. 5821). Entre su heterogéneo contenido, encontramos temas como hemerotecas, archivos, librerías especializadas o fototecas (Tapia, 2001, p. 241).

Al mismo tiempo, se estaba produciendo en los años sesenta del siglo XX un importante desarrollo de la Documentación en España, debido a los avances en nuevas tecnologías para el procesamiento, almacenamiento y difusión de información. Prueba de ello es la creación en 1967 del primer centro de documentación televisivo, el de Televisión Española — primer canal de televisión del país, que había iniciado sus emisiones en 1956-. Muchos otros medios de comunicación españoles, básicamente diarios impresos, abrieron archivos de prensa durante los años setenta, como El Correo de Andalucía, en 1975, o El País, en 1976 (desde su fundación), entre otros.

Además, y debido al éxito que estaba cosechando la Escuela Oficial de Periodismo ubicada en Madrid, otras escuelas oficiales de periodismo habían abierto durante el franquismo en España: una en Barcelona, en 1952, y otra en La Laguna, en 1964. Según Tapia (2001, pp. 241-248), se hacían alusiones a la Documentación en todos los programas de los diez cursos impartidos en 1952 en la primera de estas dos nuevas escuelas.

Pero, más allá de estos centros oficiales, otras escuelas de periodismo fueron también abiertas por iniciativas privadas y religiosas en los años cincuenta y sesenta, destacando el Instituto de Periodismo de Navarra, en Pamplona, en 1958, y la Escuela de Periodismo de la Iglesia, en Madrid, en 1960 (Real, 2012, p. 121). La Documentación también estaría presente en los planes de estudios de estos dos últimos centros formativos. En el caso del primero, como disciplina específica Documentación, englobada dentro de las disciplinas técnicas, en las que se estructuraban sus estudios junto con las culturales (Gordón, 1991, pp. 80-81). Y en el caso de la segunda, de un modo similar al ya expuesto en relación con las escuelas oficiales de periodismo, a cuyos planes de estudios se adaptó esta escuela de la Iglesia (Vigil y Vázquez, 1987, p. 78).

\section{La consolidación de la Documentación en los estudios universitarios de Periodismo}

En 1970, la Ley General de Educación reguló la incorporación de los estudios de periodismo a las universidades españolas (BOE, 1970, p. 12544) y un decreto de 1971 reguló la creación de facultades universitarias legalmente reconocidas para estudios sobre medios de comunicación, con unas enseñanzas de cinco años de Ciencias de la Información estructuradas en dos etapas - una primera más general de tres años y una segunda más específica de dos años -, así como tres "Secciones o Ramas" (que contarían, cada una, con un Departamento propio): Periodismo, Ciencias de la Imagen Visual y Auditiva y Publicidad (BOE, 1971, p. 14944). En ese mismo año, dos nuevas facultades abrieron en España: la Facultad de Ciencias de la Información de la Universidad Complutense de Madrid y la Facultad de Ciencias de la Información de la Universidad Autónoma de Barcelona (ANECA, 2007, p. 100). Estos nuevos centros universitarios reemplazaron a las escuelas oficiales de periodismo, que desaparecieron en diciembre de 1975. Al mismo tiempo, el Instituto de Periodismo de Navarra se convertiría en la ya legalmente reconocida Facultad de Ciencias de la Información de la Universidad de Navarra (de carácter privado) en 1971 (Barrera, 2002, pp. 7-8).

Asignaturas específicas relacionadas con la Documentación fueron ofertadas a los estudiantes de estas nuevas enseñanzas universitarias. No obstante, desde 1971 hasta 1974 se produjeron anualmente algunos cambios en los planes de 
estudio y las asignaturas de Documentación eran aquellas heredadas de las escuelas de periodismo no universitarias, mientras que en el primer plan de estudios universitarios de periodismo de larga duración, diseñado por el Ministerio de Educación y Ciencia para la Sección Periodismo de Ciencias de la Información impartida desde 1975 en la Facultad de Ciencias de la Información de la Universidad Complutense de Madrid, sí se incluyó un nuevo curso obligatorio titulado Documentación, ofertado en el quinto y último año (BOE, 1975, p. 22123). Para López Yepes (2002, p. 2), a partir de que devenga en estudio en el marco de este plan universitario de 1975, "la documentación informativa se convierte en objeto de estudio científico en su sentido más real".

Por otro lado, la Escuela de Periodismo de la Iglesia que se había creado en Madrid en 1960 es relevada en 1975 por el Centro de Enseñanza Superior San Pablo CEU (que pasará a denominarse en 1993 Universidad San Pablo CEU, de carácter privado). $Y$ nuevas facultades de Ciencias de la Información con estudios de periodismo nacieron en otras universidades españolas durante la década de los ochenta: la Universidad del País Vasco (en 1981) -en cuya Facultad de Ciencias de la Información se convierte la Unidad de Ciencias de la Información, de 1977, que pertenecía a la entonces denominada Universidad de Bilbao y que era dependiente de la Universidad Autónoma de Barcelona (Ronco López, 2001, p. 749)—, la Universidad Pontificia de Salamanca (de carácter privado, en 1988), la Universidad de La Laguna (en 1989) o la Universidad de Sevilla (en 1989).

En los estudios de periodismo de estos nuevos centros universitarios se impartieron cursos vinculados a la Documentación, aunque en muy diversas formas. Así, en la Universidad del País Vasco, se ofertó, en el quinto y último curso del nuevo plan de estudios de su Facultad de Ciencias de la Información aprobado en 1982, dentro de la Sección Periodismo (y también en su alternativa Publicidad) la asignatura optativa Documentación (BOE, 1983, p. 777). En la Universidad de Sevilla, la asignatura Documentación, de 120 horas, figuraba como optativa en la Sección Periodismo (también en la de Publicidad y no así en la de Imagen Visual y Auditiva) de la Licenciatura en Ciencias de la Información, de dos años (BOE, 1990, pp. 2910-2912). En la Universidad de la Laguna, sin embargo, se impartió como obligatoria, de 80 horas, Documentación Periodística, dentro del plan de estudios de segundo ciclo (Rama Periodismo) de su Facultad de Ciencias de la Información (BOE, 1989, pp. 27296-27298).

Debido a este auge de los estudios de periodismo y a las divergencias entre ellos, se produjo un control a nivel nacional y una homogeneización de los distintos planes de estudios universitarios sobre medios de comunicación social en todas estas universidades existentes en 1991 que los ofertaban (Real, 2012, p. 122), regulándose tres licenciaturas diferentes, todas de cinco años, en: Periodismo, Comunicación Audiovisual y Publicidad y Relaciones Públicas. En las tres se incluyó una asignatura troncal de 60 horas (30 teóricas y 30 prácticas), dentro del primer ciclo, denominada Documentación Informativa (BOE, 1991, p. 32903).

Además, a partir de 1991, nuevas universidades comenzaron a ofertar esta licenciatura en Periodismo: la Universidad de Santiago de Compostela (desde 1991), la Universidad Pompeu Fabra y la Universidad de Málaga (ambas desde 1992), la Universidad Carlos III de Madrid (desde 1996), la Universidad de Valencia y la Universidad Rey Juan Carlos (ambas desde 2000). Estos nuevos estudios incorporaron asignaturas específicas de Documentación dentro de sus planes de estudios, aunque no de un modo idéntico. Así, por ejemplo, tanto la Universidad de Valencia (BOE, 2000b, pp. 35166-35177) como la Rey Juan Carlos (BOE, 2000a, pp. 31239-31247) ofertaron en sus licenciaturas en periodismo una única materia vinculada al área documental, como troncal de segundo curso y 60 horas, denominada Documentación Informativa. Sin embargo, en la Universidad Carlos III de Madrid, cuya licenciatura era de segundo ciclo, se incluía como obligatoria de 60 horas, en el primero de sus dos cursos académicos, la asignatura denominada Documentación y Fuentes Informativas (BOE, 1996, pp. 30276-30289).

Al mismo tiempo que aumentaba la oferta académica periodística, las tres primeras cadenas de televisión privadas comenzaron sus emisiones en España entre 1989 y 1990, a lo que se suma el hecho de que las cadenas públicas regionales televisivas habían ya sido creadas durante los años ochenta. Así, las posibilidades de encontrar un puesto de trabajo se incrementaron enormemente para los estudiantes que finalizaban sus estudios universitarios de periodismo.

A pesar de las muchas semejanzas entre todos los nuevos estudios surgidos, ya regulados, existían algunas particularidades o diferencias en el plan de estudios ofertado por cada una de estas universidades a sus alumnos de periodismo, existiendo por tanto un cierto margen de autonomía para los centros. Así, por ejemplo, la Universidad de Sevilla ofrecería desde 1993 una licenciatura en periodismo de cuatro años, con no solo una - como la mayoría de universidades españolas - sino dos asignaturas obligatorias específicas, cada una de 45 horas de formación, vinculadas a la disciplina de la Documentación: 
Documentación Informativa, más general (2), en segundo curso, y Documentación Periodística, más específica, en cuarto y último curso (BOE, 1993, pp. 31281-31283). Sin embargo, la Universidad Complutense de Madrid, que renovó su plan de estudios de la licenciatura en periodismo en 1995, continuando esta estructurada en cinco cursos, pasó a ofertar en ella Documentación como asignatura obligatoria de 60 horas en tercer curso y Documentación Periodística y Regulación Jurídica de la Documentación como dos asignaturas optativas de segundo ciclo y de 40 horas cada una (BOE, 1995, pp. 3-15).

Más adelante, en 2002, se produjo una nueva configuración de los planes de estudios universitarios de periodismo, caracterizados por unos contenidos menos teóricos y genéricos, con semejanzas nacionales reguladas pero también particularidades en determinados centros universitarios. Además, más universidades españolas ofertarían una licenciatura en periodismo a partir de ese año: la Universidad de Murcia (desde 2002), la Universidad de Valladolid (desde 2003), así como la Universidad Miguel Hernández (desde 2005). Todas estas universidades incluían asignaturas específicas de Documentación dentro de sus planes de estudios de periodismo, aunque de un modo diferente. Así, en la titulación de segundo ciclo y dos cursos académicos ofertada por la Universidad de Murcia, aparecen tres asignaturas optativas de 50 horas y segundo curso vinculadas a la Documentación: Construcción de Servicios de Información Digital, Sistemas de Información Digital y Sistemas de Almacenamiento y Recuperación en los Medios de Comunicación Social (BOE, 2003a, pp. 42654272). Sin embargo, en la titulación de la Universidad de Valladolid se incorporaba una asignatura troncal de segundo curso y 60 horas, Documentación Informativa, y también una optativa de 60 disponible durante el segundo ciclo: Sistemas de Documentación Periodística (BOE, 2003b, pp. 41599-41604). Y en el caso de la Universidad Miguel Hernández sólo se ofertaba una asignatura de Documentación, en primer curso, de carácter troncal y de 60 horas: Documentación Informativa (BOE, 2005, pp. 16957-16962).

Por otro lado, una universidad ya existente como la Universidad Complutense de Madrid incrementó, en su nuevo plan de estudios de 2002, en 30 horas - de 60 a 90 - la asignatura obligatoria Documentación Informativa e incorporó, en el primer ciclo, una nueva asignatura optativa de 45 horas, Documentación Jurídica, a las dos ya existentes Documentación Periodística y Documentación Jurídica, de segundo ciclo y 45 horas, que se mantuvieron en el nuevo plan (BOE, 2002, pp. 26034 y 26039 ).
En definitiva, podemos deducir que la Documentación vive en España en estos años, especialmente en la última década del siglo $X X$ y en los primeros años del nuevo siglo, un momento de máximo esplendor en la historia de los estudios de periodismo, dada su gran presencia en ellos (al menos nunca antes vista en semejante grado).

\section{El declive de la Documentación en los estudios universitarios de Periodismo}

Entre 2008 y 2010 , se produjo un importante cambio en los planes de estudios universitarios en España, entre ellos los de periodismo (BOE, 2007), siendo estos adaptados obligatoriamente al Espacio Europeo de Educación Superior (EEES) en las 35 universidades españolas (públicas y privadas) que en aquel momento ofertaban estudios de periodismo (Sánchez García, 2016, p. 88). Desde entonces, todas las carreras universitarias en España, bajo la denominación de grados en lugar de licenciaturas, tendrían cuatro años de duración y 240 créditos ECTS. Además, habría una mayor diversidad de cursos en ellas, más asignaturas semestrales en detrimento de las anuales y las universidades serían más autónomas en el diseño de sus propios planes de estudio (ibidem, p. 135). También se produjo una reducción de contenidos multidisciplinares, propios de las Ciencias Sociales y Humanidades, y una inclusión de más contenidos relacionados con la práctica profesional del periodismo (ibidem, p. 138).

Pero esta nueva confección educativa supuso un duro revés y el inicio de una fase de declive para la disciplina de la Documentación dentro de los nuevos planes de estudios de periodismo $-\mathrm{y}$, en general, de todas aquellas carreras relacionadas con los medios de comunicación-, pese a las recomendaciones, acerca de reforzar los lazos entre estos dos ámbitos en lo formativo, de autores como Baranda del Campo (2012, pp. 101 y 115; 2014, p. 16) o Rojas González (2015, p. 109). Y es que estos nuevos planes de periodismo trajeron consigo una reducción considerable tanto en el número de asignaturas vinculadas a la Documentación ofertadas como en el número de horas de formación, especialmente de carácter básico y obligatorio.

Así, como ejemplo significativo de esta pérdida de peso a la que hacemos alusión, en la Facultad de Comunicación de la Universidad de Sevilla, las dos asignaturas obligatorias Documentación Informativa (de 60 horas de formación) y Documentación Periodística (de 45 horas) ofertadas en la antigua licenciatura en periodismo pasaron a una asignatura obligatoria de 60 horas, Documentación Periodística, y a una optativa de 60 
horas: Recursos Documentales Periodísticos. Además, cabe apuntar como detalle (aunque nos alejemos excepcionalmente de nuestro principal objetivo, el periodístico, en esta investigación), que las dos asignaturas obligatorias ofertadas dentro del Grado en Comunicación Audiovisual (Documentación Informativa y Documentación Audiovisual, que sumaban un total de 105 horas de formación) pasaron a una única asignatura opcional de 60 horas: Documentación Audiovisual. Y las dos asignaturas estudiadas dentro del Grado en Publicidad y Relaciones Públicas - la obligatoria Documentación Informativa, de 60 horas, y la optativa Documentación Publicitaria, de 45 horas - desaparecieron, extinguiéndose por completo la presencia de materias de Documentación en dicho grado-.

Otro claro ejemplo de esta drástica reducción lo encontramos en el Grado en Periodismo impartido en la Universidad Complutense de Madrid: de 225 a 60 horas de formación en Documentación y de cuatro asignaturas específicas de Documentación - una obligatoria y tres optativas en su plan de estudios de 2002- a solo una obligatoria en el plan de 2010 (aún vigente hoy), denominada Documentación Informativa e impartida en primer curso.

Por otro lado, desde la aprobación de estos últimos planes de estudios universitarios de Periodismo en España, el Grado en Periodismo comenzó a ofertarse también en la Universidad de Zaragoza (desde 2008), en la Universidad Jaume I y la Universidad Rovira I Virgili (en ambas desde 2009), así como en la Universidad de Castilla-La Mancha (desde 2010) y, por último, en la Universidad de Extremadura (desde 2018). Excepto en el caso de la Universidad Rovira i Virgili —la cual solo ofrecía la asignatura optativa de 60 horas Documentación para los Medios de Comunicación-, el resto de estas universidades ofertaban la obligatoria Documentación Informativa dentro de sus planes de estudios de periodismo.

No obstante, mientras que en las universidades zaragozana, manchega y en la castellonense Jaume I esta asignatura contaba con 60 horas de formación, la universidad extremeña, en una muestra de su interés y apuesta por la Documentación, duplicó la duración de este curso, ampliándolo a 120 horas obligatorias, añadiendo además en su oferta educativa la asignatura optativa Fuentes de información para periodismo, de 60 horas (aunque esta aún no se ha impartido nunca, dado que, al ser de cuarto curso, la materialización del nuevo plan de estudios extremeño no ha llegado aún a este nivel).

En la actualidad, existen 19 universidades públicas en España que imparten el Grado en
Periodismo. En todos sus planes de estudios vigentes para el curso académico 2020/2021 se ofrece al menos un curso específico de Documentación a sus estudiantes y futuros periodistas, con 60 horas de formación. No obstante, se aprecian diferencias sustanciales entre estas distintas ofertas educativas específicas (Tabla I, en la página siguiente).

Así, en cuatro de estas universidades se puede estudiar más de una asignatura específica vinculada a esta disciplina, aunque también con divergencias notorias entre ellas. De este modo, en la Universidad de Murcia pueden cursarse hasta tres materias -Documentación en los Medios de Comunicación Social I, Documentación en los Medios de Comunicación Social ll y Construcción de Servicios de Información Digital_-, todas ellas de formación básica u obligatoria y que suman hasta 240 horas totales, posicionándose así este centro como el que proporciona en la actualidad una mayor formación específica de Documentación dentro del Grado en Periodismo.

En un segundo peldaño se encontraría la Universidad Miguel Hernández, al formar en Documentación a sus alumnos de periodismo con 120 horas, a través de dos asignaturas obligatorias o de formación básica, de 60 horas cada una - Gestión de Bases de Datos y Documentación Informativa - Tras este centro, se hallarían, en un tercer escalón, las dos universidades que ofertan 60 horas de formación obligatoria o básica y 60 opcionales: la Universidad de Sevilla (Documentación Periodística y Recursos Documentales y Periodismo de Datos, respectivamente) y la de Extremadura (Documentación Informativa y Fuentes de Información para Periodismo, respectivamente). Y en un cuarto eslabón, se encontraría la Universidad Carlos III de Madrid, que brinda a sus alumnos una formación básica de 30 horas a través de la asignatura Técnicas de Búsqueda y Uso de la Información y les ofrece también la materia optativa de 60 horas Documentación Digital.

A continuación, y en un quinto nivel, estarían las doce universidades públicas que forman en Documentación a sus alumnos del grado en periodismo a través de una única asignatura de carácter básico u obligatorio de 60 horas. De ellas, nueve lo hacen bajo la denominación de Documentación Informativa: las universidades de Santiago de Compostela (la única de estas que la oferta como formación básica en lugar de obligatoria), Complutense de Madrid, Castilla-La Mancha, Jaume I, La Laguna, Málaga, Rey Juan Carlos, Valladolid y Zaragoza. Las otras tres universidades dan a esta única materia de 60 horas otras denominaciones, como Documentación Comunicativa (básica) en el caso de la Universidad de Valencia, Formatos Digitales $y$ 
Documentación (básica) en la Universidad Pompeu Fabra o Gestión de Fuentes Documentales (obligatoria) en la Universidad del País Vasco.

Por último, encontraríamos las dos universidades públicas restantes que carecen en sus grados en periodismo de asignaturas de formación obliga- toria o básica vinculadas a la Documentación, pero que sí ofrecen en su plan de estudios una asignatura optativa de 60 horas relacionada con dicha área: la Universidad Autónoma de BarceIona (materia Documentación Periodística) y la Universidad Rovira i Virgili (Documentación para los Medios de Comunicación).

\begin{tabular}{|c|c|c|c|c|}
\hline $\begin{array}{l}\text { Universidad pública española } \\
\text { con Grado en Periodismo }\end{array}$ & $\begin{array}{l}\text { Asignaturas específicas vinculadas } \\
\text { a la Documentación }\end{array}$ & $\begin{array}{l}N^{\circ} \text { de } \\
\text { Horas }\end{array}$ & $\begin{array}{l}\text { Tipo de } \\
\text { asignatura }\end{array}$ & Curso \\
\hline \multirow[t]{3}{*}{ Universidad de Murcia } & $\begin{array}{l}\text { Documentación en los Medios de } \\
\text { Comunicación Social I }\end{array}$ & 60 & $\begin{array}{l}\text { Formación } \\
\text { básica }\end{array}$ & $1^{\circ}$ \\
\hline & $\begin{array}{l}\text { Documentación en los Medios de } \\
\text { Comunicación Social II }\end{array}$ & 60 & $\begin{array}{l}\text { Formación } \\
\text { básica }\end{array}$ & $2^{\circ}$ \\
\hline & $\begin{array}{l}\text { Construcción de Servicios de Información } \\
\text { Digital }\end{array}$ & 120 & Obligatoria & $4^{\circ}$ \\
\hline \multirow[t]{2}{*}{ Universidad Miguel Hernández } & Gestión de bases de datos & 60 & $\begin{array}{l}\text { Formación } \\
\text { básica }\end{array}$ & $1^{0}$ \\
\hline & Documentación Informativa & 60 & Obligatoria & $2^{\circ}$ \\
\hline \multirow[t]{2}{*}{ Universidad de Sevilla } & Documentación Periodística & 60 & Obligatoria & $2^{\circ}$ \\
\hline & Recursos Documentales y Periodismo de Datos & 60 & Optativa & $3^{\circ}$ \\
\hline \multirow[t]{2}{*}{ Universidad de Extremadura } & Documentación Informativa & 60 & Obligatoria & $3^{\circ}$ \\
\hline & Fuentes de información para periodismo & 60 & Optativa & $4^{\circ}$ \\
\hline \multirow[t]{2}{*}{ Universidad Carlos III de Madrid } & Técnicas de búsqueda y uso de la información & 30 & $\begin{array}{l}\text { Formación } \\
\text { básica }\end{array}$ & $1^{\circ}$ \\
\hline & Documentación Digital & 60 & Optativa & $3^{\circ}$ \\
\hline Universidad de Valencia & Documentación comunicativa & 60 & $\begin{array}{l}\text { Formación } \\
\text { básica }\end{array}$ & $1^{\circ}$ \\
\hline $\begin{array}{l}\text { Universidad de Santiago de } \\
\text { Compostela }\end{array}$ & Documentación Informativa & 60 & $\begin{array}{l}\text { Formación } \\
\text { básica }\end{array}$ & $2^{\circ}$ \\
\hline $\begin{array}{l}\text { Universidad Complutense de } \\
\text { Madrid }\end{array}$ & Documentación Informativa & 60 & Obligatoria & $1^{\circ}$ \\
\hline Universidad de CastillaLa Mancha & Documentación Informativa & 60 & Obligatoria & $2^{\circ}$ \\
\hline Universitat Jaume I & Documentación Informativa & 60 & Obligatoria & $1^{\circ}$ \\
\hline Universidad de La Laguna & Documentación Informativa & 60 & Obligatoria & $2^{\circ}$ \\
\hline Universidad de Málaga & Documentación Informativa & 60 & Obligatoria & $2^{\circ}$ \\
\hline Universidad Rey Juan Carlos & Documentación Informativa & 60 & Obligatoria & $2^{\circ}$ \\
\hline Universidad de Valladolid & Documentación Informativa & 60 & Obligatoria & $2^{\circ}$ \\
\hline Universidad de Zaragoza & Documentación Informativa & 60 & Obligatoria & $1^{\circ}$ \\
\hline Universidad Pompeu Fabra & Formatos Digitales y Documentación & 60 & $\begin{array}{l}\text { Formación } \\
\text { básica }\end{array}$ & $2^{\circ}$ \\
\hline Universidad del País Vasco & Gestión de fuentes documentales & 60 & Obligatoria & $2^{\circ}$ \\
\hline $\begin{array}{l}\text { Universidad Autónoma de } \\
\text { Barcelona }\end{array}$ & Documentación Periodística & 60 & Optativa & $3^{\circ}$ \\
\hline Universidad Rovira i Virgili & $\begin{array}{l}\text { Documentación para los Medios de } \\
\text { Comunicación }\end{array}$ & 60 & Optativa & $4^{\circ}$ \\
\hline
\end{tabular}

Tabla I. Asignaturas de Documentación en los Grados en Periodismo de las universidades públicas españolas en el curso 2020/2021 docencia de la Documentación en los estudios de Periodismo. // lbersid. 15:2 (jul.-dic. 2021) 57-65. 


\section{Conclusiones}

Gracias a la importancia que la disciplina de la Documentación ha tenido históricamente en las escuelas españolas de periodismo desde el origen de estas en 1926, tanto en estudios preuniversitarios como sobre todo universitarios (desde 1971), la Documentación Periodística e Informativa ha adquirido un peso considerable en nuestro país y se ha ido dotando progresivamente de un corpus doctrinal importante a partir de los años setenta del siglo $X X$.

No obstante, pese al período de esplendor vivido en cuanto a presencia de la docencia de la Documentación en los planes de estudios universitarios de periodismo, especialmente en la última década del siglo $X X$ y en casi toda la primera del $\mathrm{XXI}$, la adaptación de los estudios universitarios al Espacio Europeo de Educación Superior ha supuesto, desde finales de la primera década del presente siglo, una reducción progresiva, aún vigente, de asignaturas y horas de formación en Documentación en estos estudios, especialmente de carácter básico y obligatorio. Además, en la actualidad, hemos observado diferencias considerables a nivel nacional entre las ofertas de asignaturas vinculadas a la Documentación dentro del Grado en Periodismo impartido en las universidades públicas españolas, oscilando estas materias entre tres y una, con disparidad en cuanto al número de horas de formación y al carácter de estas, así como en lo que respecta al curso o año en el que se programan (desde primero hasta cuarto).

Cabe apuntar que esta progresiva pérdida de peso de la Documentación en los estudios universitarios de Periodismo (y, en general, en estudios relacionados con los medios de comunicación), transcurre en paralelo con una drástica disminución en el número y dimensiones de los centros de documentación de empresas periodísticas en los últimos años (Melendez-Malavé y Hirschfeld-Suárez, 2016, p. 612; Marcos Recio y Edo, 2015, p. 420), lo que debería, todo ello, hacernos reflexionar y mantenernos en alerta a los educadores, investigadores y profesionales.

$Y$ es que si los futuros periodistas y editores, que son los estudiantes de periodismo de hoy, pierden su interés o dejan de recibir una formación adecuada en este campo, podrían presentarse enormes dificultades para una búsqueda y recuperación eficaz y eficiente de las fuentes periodísticas, derivadas de una deficiente o inadecuada gestión documental. Del mismo modo, podría ponerse en peligro el patrimonio documental periodístico español —diarios y revistas impresas y digitales, fotografías de prensa, emisiones de radio y televisión, etc.- - de un valor testimonial e histórico incalculable para las futuras generaciones.

\section{Notas}

(1) Aunque el Instituto de Periodismo de Navarra ha sido parte de la Universidad de Navarra desde 1958, este no fue reconocido como una facultad universitaria de periodismo dentro del marco legal español hasta el 8 de noviembre de 1971 (Barrera, 2002). Más bien fue una "facultad presentida" (Vigil y Vázquez, 1987, p. 153).

(2) El concepto más general Documentación Informativa se entendió inicialmente en España como "aquella parte de la Documentación que tiene por objeto el estudio del proceso de transmisión de las fuentes para la obtención de nuevo conocimiento en la investigación de la comunicación social y en el trabajo en el seno de la empresa informativa" (López Yepes, 1981, p. 23), mientras que la Documentación Periodística se concibió como una "parte de la Documentación Informativa que estudia el proceso de transmisión de las fuentes para la obtención de nuevo conocimiento que sea de utilidad para la investigación periodística, refiriéndose tanto a la documentación en medios impresos, sonoros como audiovisuales" (López Yepes y Ros García, 1993, pp. 129-131).

\section{Referencias}

Agencia Nacional de Evaluación de la Calidad y Acreditación (ANECA) (2007). Libro blanco de los Títulos de Grado en Ciencias de la Comunicación. ANECA. https://n9.cl/ 62qxq (2021-02-24).

Baranda del Campo, C. (2012). La barrera entre el periodista y el documentalista. // Documentación De Las Ciencias De La Información. 35 (Diciembre 2012) 101-118.

Baranda del Campo, C. (2014). El espacio común entre dos disciplinas: Periodismo y Documentación. Cuadernos De Documentación Multimedia. 25 (Diciembre 2014) 2-17.

Barrera, Carlos (2002). Notas para una historia del Instituto de Periodismo de la Universidad de Navarra. // Communication \& Society. 15:1 (2002) 7-38.

Barrera, Carlos; Vaz, Aires (2003). The Spanish Case: A Recent Academic Tradition. // Frohlich, R.; Holtz-Bacha, C. Journalism Education in Europe and North America: An International Comparison. Cresskill, NJ: Hampton Press.

Boletín Oficial del Estado (BOE) (1967). 106 (4 de mayo) 5821-5822. https://n9.cl/u7pkb (2020-12-09).

Boletín Oficial del Estado (BOE) (1970). 187 (6 de agosto) 12525-12546. https://n9.cl/9zte (2020-12-10).

Boletín Oficial del Estado (BOE) (1971). 220 (14 de septiembre) 14944-14945. https://n9.cl/lfwba (2020-12-10).

Boletín Oficial del Estado (BOE) (1975). 252 (21 de octubre) 22122-22123. https://n9.cl/q6ztr (2020-12-11).

Boletín Oficial del Estado (BOE) (1983). 10 (12 de enero) 777. https://n9.cl/zaizj (2020-12-15).

Boletín Oficial del Estado (BOE) (1989). 202 (24 de agosto) 27296-27298. https://n9.cl/p4eix (2020-12-16).

Boletín Oficial del Estado (BOE) (1990). 26 (30 de enero) 2910-2912. https://n9.cl/2jit9 (2020-12-17).

Boletín Oficial del Estado (BOE) (1991). 243 (10 de octubre) 32902-32904. https://n9.cl/hde0 (2021-01-17).

Boletín Oficial del Estado (BOE) (1993). 265 (5 de noviembre) 31281-31289. https://n9.cl/qrps (2020-12-18).

Boletín Oficial del Estado (BOE) (1995). Suplemento 239 (6 de octubre) 3-15. https://n9.cl/4542c (2020-12-20).

Boletín Oficial del Estado (BOE) (1996). 244 (9 de octubre) 30276-30289. https://n9.cl/ey19l (2020-12-21). 
Boletín Oficial del Estado (BOE) (2000a). 215 (7 de septiembre) 31239-31247. https://n9.cl/kaje5 (2021-01-12).

Boletín Oficial del Estado (BOE) (2000b). 246 (13 de octubre) 35166-35177. https://n9.cl/ejni5 (2021-01-18).

Boletín Oficial del Estado (BOE) (2002). 169 (16 de julio) 26033-26048. https://n9.cl/1afk6 (2021-01-20).

Boletín Oficial del Estado (BOE) (2003a). 27 (31 de enero) 4265-4272. https://n9.cl/jvt0u (2021-01-23).

Boletín Oficial del Estado (BOE) (2003b). 281 (24 de noviembre) 41599-41604. https://n9.cl/litfg (2021-01-25).

Boletín Oficial del Estado (BOE) (2005). 119 (19 de mayo) 16957-16962. https://n9.cl/bj9pq (2021-02-07)

Boletín Oficial del Estado (BOE) (2007). 260 (30 de octubre) 44037-44048. https://n9.cl/uwary (2021-02-13).

Cantavella, Juan (2017). La Escuela de Periodismo de EI Debate. Madrid: CEU y Ediciones APM.

Gordón Pérez, Mercedes (1991). La Enseñanza del Periodismo en el Mundo Occidental. Estudio Histórico y Comparado de tres Escuelas. Madrid: Editorial de la Universidad Complutense. Colección Tesis Doctorales. $\mathrm{N}^{\circ}$ 387/91.

Graña González, Manuel (1930). La Escuela de Periodismo: programas y métodos. Madrid: Compañía Ibero-Americana de Publicaciones.

López Yepes, José (1978). Teoría de la Documentación. Pamplona: Eunsa.

López Yepes, José (1981). El estudio de la Documentación. Madrid: Tecnos.

López Yepes, José; Ros García, Juan (1993). ¿Qué es Documentación? Teoría e historia del concepto en España. Madrid: Síntesis.

López Yepes, J. (2002). La Documentación en la empresa informativa. Notas para el estudio de sus fundamentos. // Cuadernos De Documentación Multimedia. 12 (Julio 2002).

Marcos Recio, Juan Carlos; Concha Edo (2015). Análisis de la nueva perspectiva de la documentación periodística en los medios de comunicación españoles. // Revista General de Información y Documentación. 25:2 (Diciembre 2015) 389-423.

Meléndez-Malavé, Natalia; Hirschfeld-Suárez, Rocío (2016). Situación de los centros de documentación en los medios escritos andaluces. // El profesional de la información. 25:4 (Julio-Agosto 2016) 606-615.

Real, Elena (2012). Los estudios de periodismo en España, competencias y destrezas. // Marta Lazo, C.; Agustín Lacruz, M.C. y Ubieto Artur, M.I. (coord.). Competencias interdisciplinares para la comunicación y la información en la sociedad digital. Icono 14 editorial. 115-144.

Rojas González, X. (2015). La formación del documentalista y archivista en documentación audiovisual en Costa Rica. // Cuadernos De Documentación Multimedia. 26 (Noviembre 2015) 97-112.

Ronco López, María Milagros (2001). Aportaciones a la historia de la Facultad de Ciencias de la Información en la Universidad del País Vasco. La asignatura Documentación. // Cuadernos de Documentación Multimedia. 10 (2001) 749-755.

Sánchez-García, Pilar (2016). Los efectos de la primera fase del EEES en la enseñanza del periodismo en España: mayor especialización y formación práctica. // Communication \& Society. 29:1 (2016) 125-143.

Tapia López, Alicia (1995). Antecedentes de la documentación periodística en España. // Documentación de las Ciencias de la Información. 18 (Enero 1995) 233-244.

Tapia López, Alicia (2001). Las primeras enseñanzas de documentación en periodismo. // Documentación de las Ciencias de la Información. 24 (Enero 2001) 231-251.

Vigil y Vázquez, Manuel (1987). El periodismo enseñado. De la Escuela de "El Debate" a Ciencias de la Información. Barcelona: Mitre.

Enviado: 2021-04-06. Segunda versión: 2021-06-28. Aceptado: 2021-06-28. 
